\title{
AGILE: ten years after
}

\section{Carlotta Pittori*i}

INAF-OAR and ASI-SSDC, Roma, Italy

E-mail: carlotta.pittoriessdc.asi.it

AGILE is an Italian Space Agency (ASI) mission devoted to $\gamma$-ray astrophysics in the $30 \mathrm{MeV}$ - $50 \mathrm{GeV}$ energy range, with simultaneous X-ray imaging capability in the $18-60 \mathrm{keV}$ band. Launched in April 2007, the AGILE satellite has completed its tenth year of operations in orbit with all detectors operating nominally. The small high-tech jewel, designed for an operative life of only two years, continues its mission with high efficiency, and it is substantially contributing to improve our knowledge of the high-energy sky. We present an overview of the main AGILE $\gamma$-ray satellite scientific results and discoveries.

XII Multifrequency Behaviour of High Energy Cosmic Sources Workshop

12-17 June, 2017

Palermo, Italy

* Speaker.

$\dagger$ on behalf of the AGILE Collaboration. 


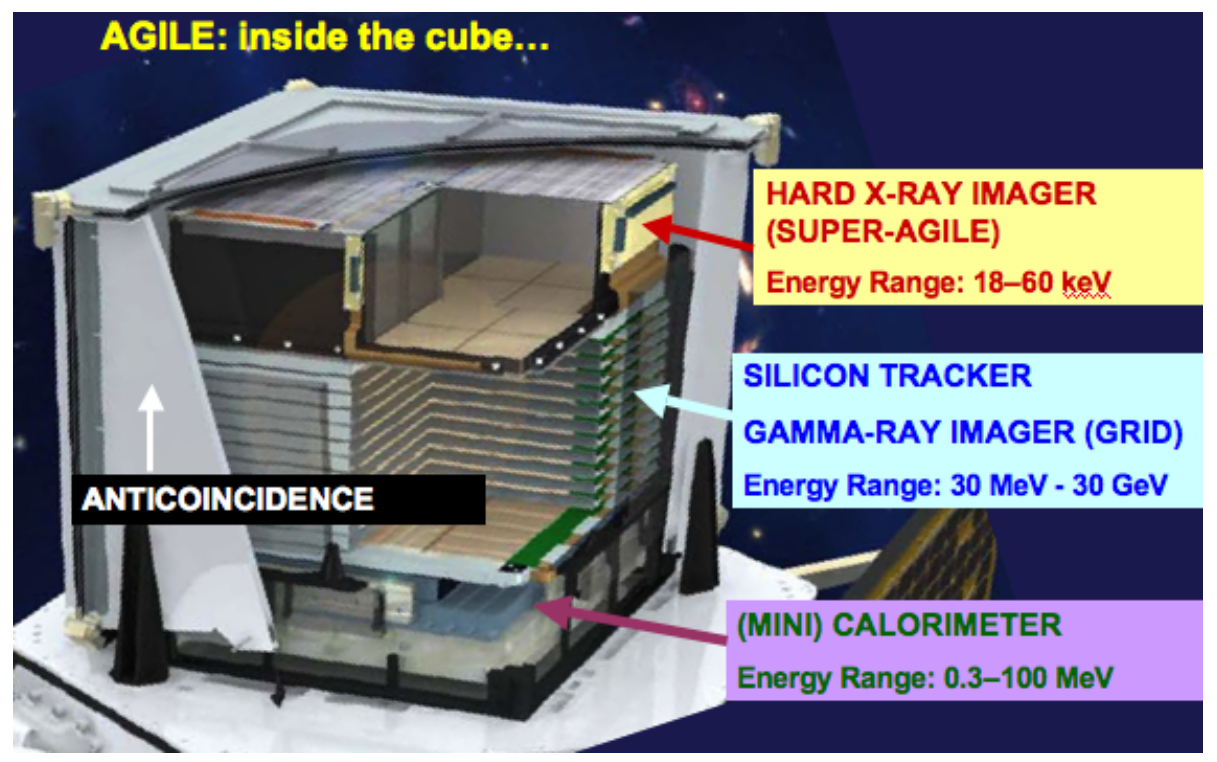

Figure 1: The AGILE payload.

\section{Introduction}

The AGILE (Astro-rivelatore Gamma a Immagini LEggero) satellite of the Italian Space Agency (ASI), is a $\gamma$-ray astrophysics mission with INAF, INFN and CIFS participation [1]. Launched on April 23, 2007, AGILE has just completed its tenth year of operations, and continues its exploration of the high-energy Universe, also giving a crucial contribution in the search of electromagnetic counterparts of gravitational waves (GW). The AGILE payload consists of three instruments (see Fig. 1): the Gamma Ray Imager Detector (GRID) sensitive in the energy range 30 MeV-30 GeV, a Mini-Calorimeter (MCAL) sensitive in the energy range $350 \mathrm{keV}$ to $100 \mathrm{MeV}$ that works both as a slave of the GRID and as an autonomous detector for transient events, and a hard X-ray imager (Super-AGILE), sensitive in the energy range 18-60 keV. An anticoincidence system (AC) of segmented plastic scintillators is used for particle background rejection.

We present here a selection of the main AGILE science highlights after ten years of operations, and some recent updates related to gravitational wave $(\mathrm{GW})$ astrophysics, and the hunt for electromagnetic counterparts.

\section{Top three AGILE discoveries}

We summarise in this section the three main AGILE discoveries in high-energy astrophysics above $100 \mathrm{MeV}$ for which the Italian satellite will be remembered. These important AGILE scientific results were subsequently confirmed also by the NASA Fermi satellite, but "credit where credit is due".

\subsection{Discovery of Crab Nebula variability}

The Crab Nebula is the remnant of a supernova explosion witnessed by Chinese astronomers in 1054 , powered by a very powerful pulsar with rotating period of $0.33 \mathrm{~ms}$. The average unpulsed 


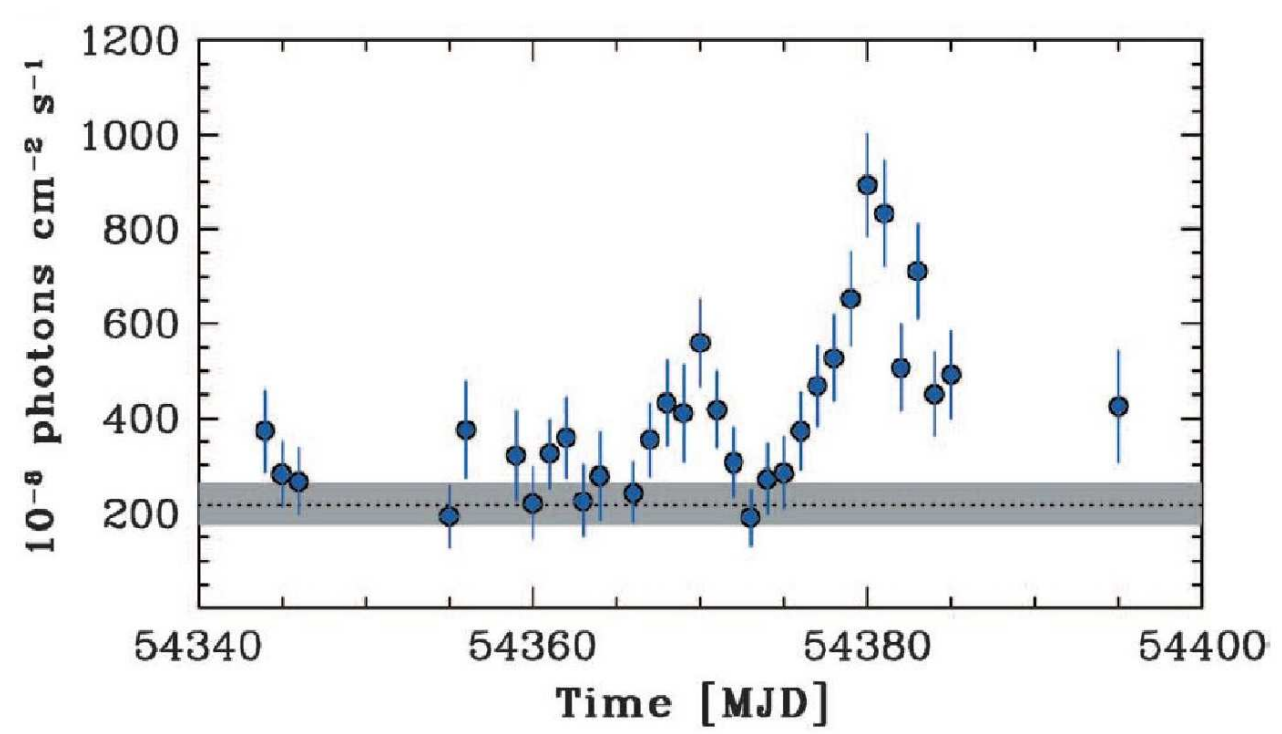

Figure 2: Crab (pulsar plus Nebula) light curve from AGILE data (E > $100 \mathrm{MeV})$ during the period 27 September - 12 October 2007 (1-day binning). The dotted line and gray band show the average Crab flux and the $3 \sigma$ uncertainty range [3].

emission from the Crab Nebula was always considered essentially stable. Crab was considered to be an almost ideal standard candle, a nearly constant source from optical to $\gamma$-ray energies, with possible long-term variations of the nebular flux over a few-year timescale.

On September 22, 2010, a strong and unexpected $\gamma$-ray flare from the Crab Nebula was announced by AGILE at energies greater than $100 \mathrm{MeV}[2,3]$. This surprising AGILE discovery was confirmed one day later by Fermi [4, 5]. AGILE had also previoulsy detected a giant flare (see Fig. 2) from the Crab in October, 2007 which was not publicly announced at the time since it occurred during the initial Science Verification Phase of the satellite. However, in the "First AGILE Catalog" paper [6] it was reported that anomalous episodic high-flux values observed from the Crab in 2007 were under investigation. We know now of several major $\gamma$-ray flares from the Crab Nebula detected by the AGILE-GRID and Fermi-LAT, up to October 2016. No optical or X-ray emission in coincidence with the $\gamma$-ray flaring has been unambiguously detected to date. In addition to the intense day-long flares detected by AGILE and Fermi, AGILE found evidence for week-long, less intense episodes of enhanced $\gamma$-ray emission that called "waves" [7].

AGILE $\gamma$-ray observations challenge standard models of nebular emission, and imply a new acceleration mechanism coming into play to induce intense and rapid flux variations in the Crab Nebula in the energy band above $100 \mathrm{MeV}$, on approximately 1-day time scales. Plasma instabilities possibly related to magnetic field reconnection in specific sites in the Nebula can be envisioned.

\subsection{Evidence of cosmic-ray acceleration in the SNR W44}

AGILE was the first to discover a clear indication of the so-called "pion bump" in the $\gamma$-ray emission from the supernova remnant (SNR) W44 [8]. W44 is an intermediate-age SNR (about 20000 years old), whose ejecta expand in a dense medium as shown by a prominent radio shell, interacting with nearby molecular clouds. The AGILE-GRID instrument reaches its optimal sensitivity just at energies between $50 \mathrm{MeV}$ and a few $\mathrm{GeV}$, and provided the first direct evidence that 


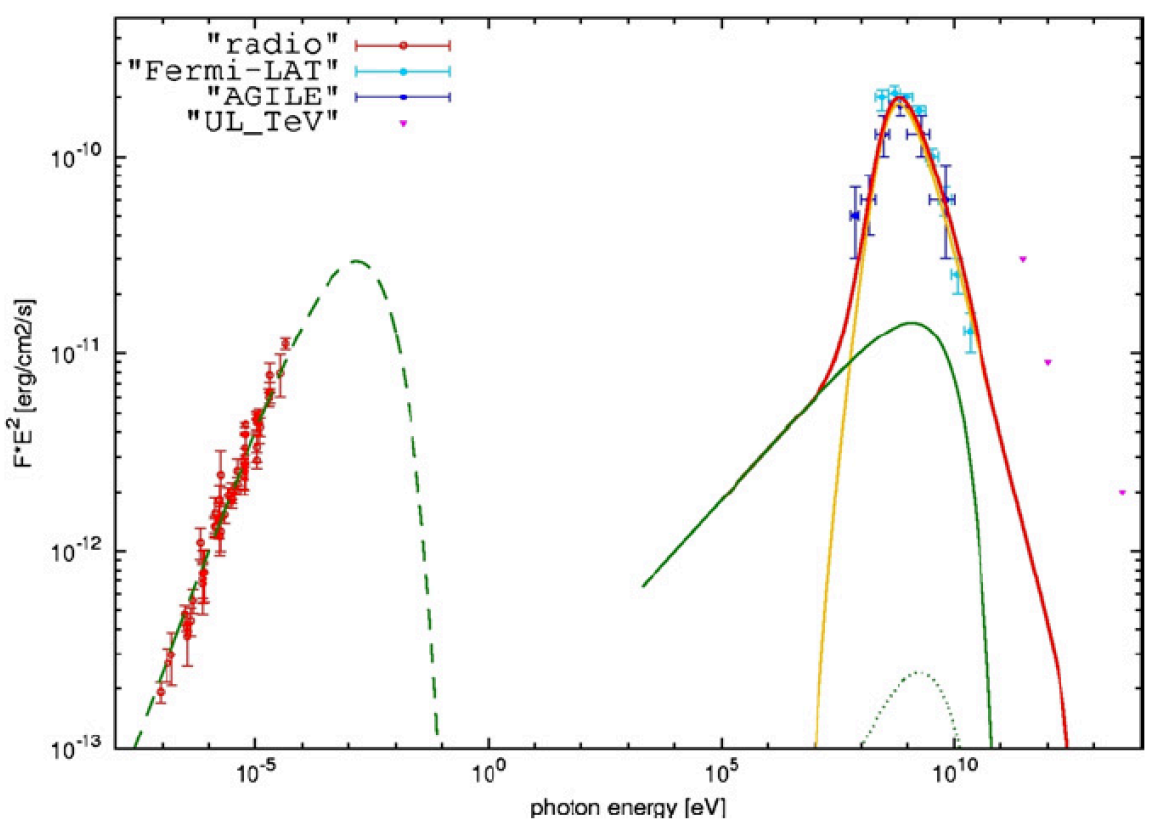

Figure 3: Spectral energy distribution of the SNR W44: radio data in red and AGILE $\gamma$-ray data in blue [8]. Fermi-LAT data (cyan) at the time of AGILE discovery were limited to the energy band above $200 \mathrm{MeV}$ [9]. The yellow curve shows the neutral pion emission from the accelerated proton distribution (see text).

hadron-dominated models are consistent with all multiwavelength constraints derived from radio, optical, X-ray, and $\gamma$-ray observations of the SNR W44.

The broadband spectrum shown in Fig. 3 is successfully modeled by the $\gamma$-ray emission resulting from the decay of neutral pions produced by accelerated protons/ions with a spectral index near 3 and a cut-off energy of about $6 \mathrm{GeV}$. This important AGILE result was later confirmed by Fermi-LAT data in [10], following an update to Fermi event classification and background rejection methods.

\subsection{Discovery of transient $\gamma$-ray emission from Cygnus X-3 microquasar}

AGILE detected for the first time several $\gamma$-ray flares above $100 \mathrm{MeV}$ from the Cygnus X-3 microquasar, in correlation with a repetitive pattern of multiwavelength radio and $\mathrm{X}$-ray emission [11]. Galactic microquasars are binary systems consisting of a neutron star or stellar-mass black hole, accreting mass from a companion star. These systems were known to produce relativistic jets, generally together with radio flares. Notably, all $\gamma$-ray flares reported by AGILE (see Fig. 4 , red arrows) occur either in coincidence with low hard-X fluxes or during transitions from low to high values of hard-X fluxes, and usually appear before major radio flares. This important AGILE discovery has been subsequently confirmed by Fermi, which was also able to identify the 4.8 hours orbital period in $\gamma$-rays, securing unambigously the temporal signature of the binary system [12].

AGILE and Fermi observations indicate that the Cygnus X-3 binary system is indeed able to accelerate particles up to relativistic energies, and to emit $\gamma$-rays above $100 \mathrm{MeV}$ challenging previous models of particle acceleration. 


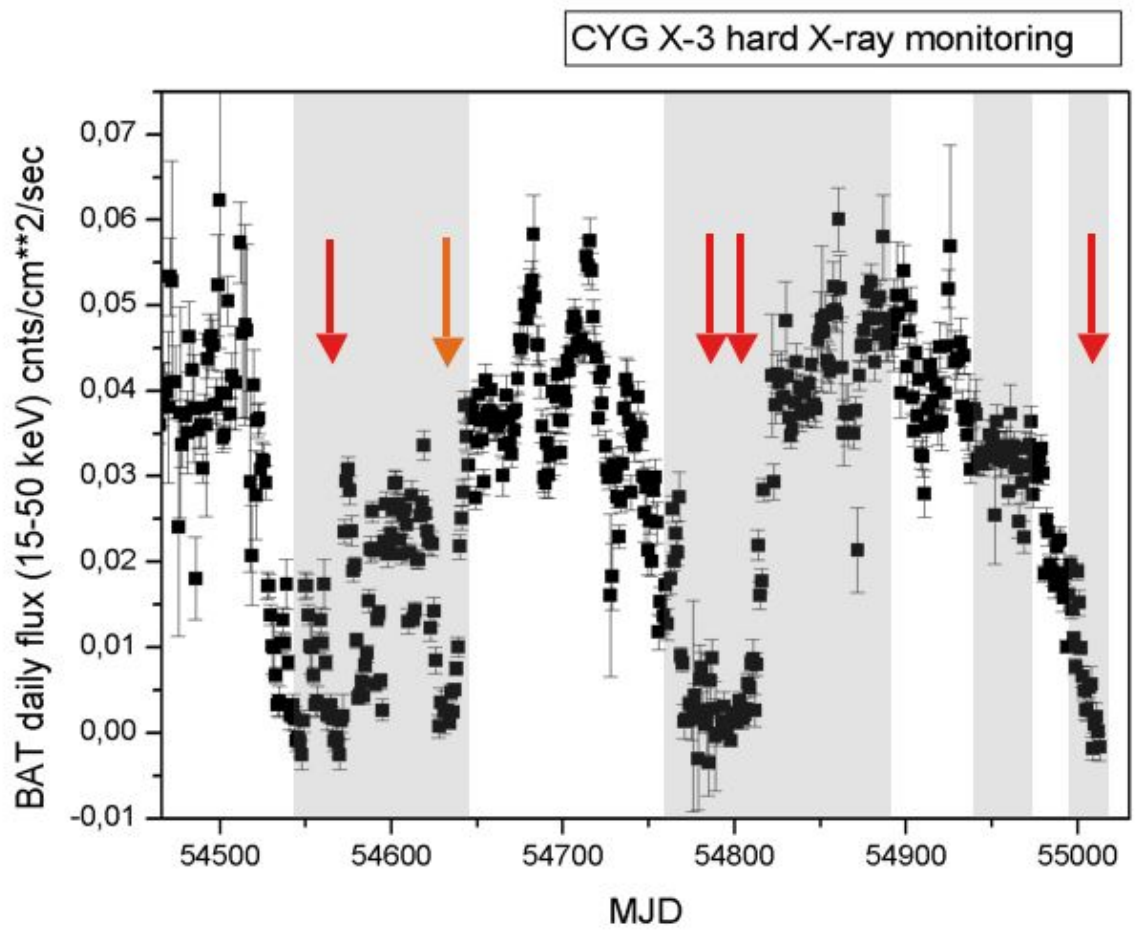

Figure 4: Swift hard-X-ray monitoring of Cygnus X-3 between January 1, 2008 and June 30, 2009. The red arrows mark the occurence of major $\gamma$-ray flares of Cygnus X-3 detected by AGILE.

\section{Other AGILE scientific highlights}

Other important scientific results for which AGILE should be remembered include:

- Vela-X: the AGILE detection the first time of $\gamma$-ray emission above $100 \mathrm{MeV}$ from a Pulsar Wind Nebula (PWN), the Vela-X PWN, as described in [13].

- $\eta$-Carinae: the first detection of a colliding wind binary system (CWB) above $100 \mathrm{MeV}$ in the $\eta$-Carinae region [14].

- Bright $\gamma$-ray flaring blazars: AGILE observations of $\gamma$-ray flares from Flat Spectrum Radio Quasars (FSRQ) such as 3C 454.3 [15], 3C 279 [16, 17], PKS 1830-211 [18], 4C +21.35 [19], together with their multi-wavelength behavior that show in general a very high Compton dominance, challenging simple one-zone leptonic theoretical models [20].

- MCW 656:: AGILE detection of a new transient source AGL J2241+4454 [21], which led to the subsequent discovery of the first ever known hidden black hole in the Be star binary system MCW 656 [22].

- Terrestrial Gamma-Ray Flashes: the AGILE Minicalorimeter detects TGFs that last a few thousandths of a second, and produce $\gamma$-ray flashes up to $100 \mathrm{MeV}$, on timescales as low as $<5 \mathrm{~ms}$ [23]. The crucial AGILE contribution to TGF science is the discovery that the TGF spectrum extends well above $40 \mathrm{MeV}$, and that the high energy tail of the TGF spectrum is harder than expected. 


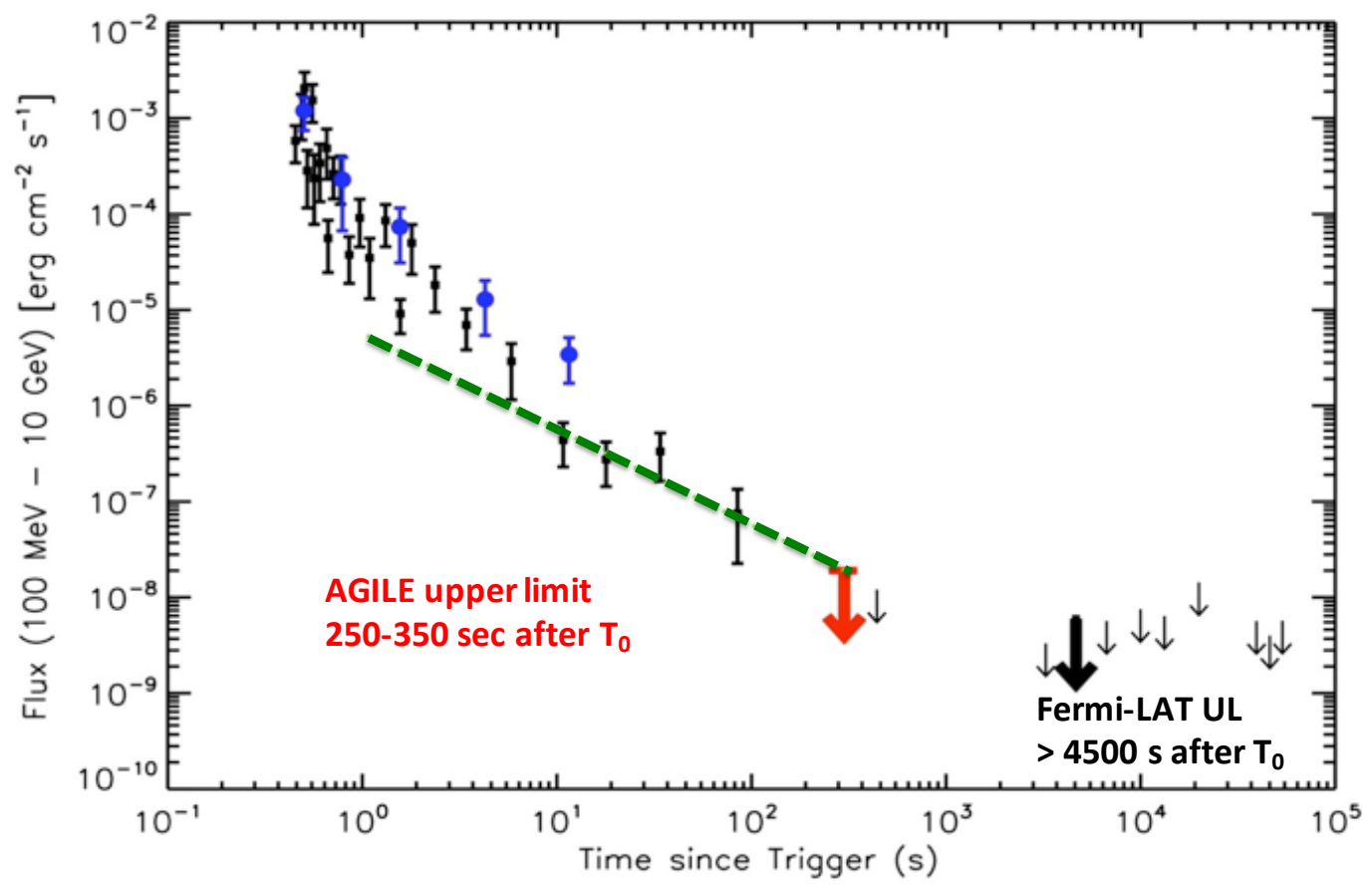

Figure 5: The AGILE (blue circles) [27] and Fermi-LAT (black squares) [28] $\gamma$-ray lightcurves of the short GRB090510 scaled in flux and time as if it originated at the GW event GW150914 luminosity distance. The AGILE-GRID upper limit to $\gamma$-ray emission above $100 \mathrm{MeV}$ is shown in red, the corresponding Fermi-LAT upper limit is marked in black.

\section{AGILE and GW astrophysics}

The recent direct detection of gravitational waves (GW) by the LIGO-Virgo Collaboration since September 14, 2015 [24] started a new era in astronomy. The very fast AGILE ground segment alert system $[25,26]$ has been recently further optimized for the search of electromagnetic counterparts of gravitational waves, allowing the AGILE Team to perform a full data reduction and the preliminary Quick Look scientific analysis only 25/30 minutes after the telemetry download from the spacecraft.

The AGILE observations have provided the fastest response and the most significant upper limits above $100 \mathrm{MeV}$ to the first detected gravitational wave event GW150914 (see Fig. 5), and to all other GW events detected up to now with optimal gamma-ray sensitivity [27, 29, 30]. We look forward for future follow-up gamma-ray observations of GW sources with AGILE.

\section{A new data analysis web-tool: AGILE-LV3}

A new web interface for official interactive on-line analysis of AGILE data was developed at the AGILE Data Center in ASI. For an easy on-line $\gamma$-ray data analysis the interested user may query the entire level 3 (LV3) public AGILE archive through the AGILE-LV3 tool ${ }^{1}$, which does not require any locally installed software or calibrations, and it has been also tested with students.

\footnotetext{
${ }^{1}$ http://www.ssdc.asi.it/mmia/index.php?mission=agilelv3mmia
} 


\section{Acknowledgments}

The author would like to acknowledge the financial support of ASI under contract to INAF: ASI 2014-049-R.0 dedicated to the ASI Space Science Data Center (SSDC, previously ASDC).

\section{References}

[1] Tavani, M., Barbiellini, G., Argan, A., et al. 2009, A.\&A., 502, 995

[2] Tavani, M. et al., 2010, ATel \#2855, NASA ADS bibliographic code: 2010ATel.2855....1T

[3] Tavani, M. et al., 2011, Science Vol. 331, 736-739

[4] Buehler, R. et al., 2010, ATel \#2861, NASA ADS bibliographic code: 2010ATel.2861....1B

[5] Abdo, A. A. et al., 2011, Science Vol. 331, 739

[6] Pittori, C. et al., 2009, A.\&A., 506, 1563

[7] Striani, E. et al., 2013 ApJ 765, 52

[8] Giuliani, A. et al., 2011, ApJ 742, L30

[9] A. A. Abdo et al., 2010, Science 327, 1103

[10] Ackermann M., et al., 2013, Science, 339, 807

[11] Tavani, M. et al., 2009, Nature 462, 620-623

[12] Abdo A. A., Ackermann M., Ajello M., et al., 2009a, Sci, 326, 1512

[13] Pellizzoni, A., Trois, A., Tavani, M., et al. 2010, Science, 327, 663

[14] Tavani, M., Sabatini, S., Pian, E. et al. 2009, ApJ, 698, L142

[15] Vercellone, S., Striani, E., Vittorini, V., et al. 2011, ApJ, 736, L38

[16] C. Pittori et al., 2018 ApJ, 856, 99

[17] Giuliani, A. et al., 2009, A. \& A., 494, 509

[18] Donnarumma, I. et al., 2011, ApJL, 736, L30

[19] Verrecchia, F., et al., 2014, ATel \#6733, NASA ADS Bibliographic code: 2014ATel.6733....1V

[20] Tavani, M., Vittorini, V., and Cavaliere, A. 2015, ApJ, 814, 51

[21] Lucarelli, F. et al., 2010, ATel \#2761, NASA ADS Bibliographic code: 2010ATel.2761....1L

[22] Munar-Adrover, P., et al. 2016, ApJ, 829, 101

[23] Marisaldi, M., et a. 2010, JGRA, 115, A00E13

[24] Abbott B.P. et al., 2016a, Phys. Rev. Letters, 116, 061102

[25] Pittori, C. 2013, Nuclear Physics B Proceedings Supplements, 239, 104

[26] Bulgarelli, A., Trifoglio, M., Gianotti, F., et al. 2014, ApJ, 781, 19

[27] Tavani, M., Pittori, C., Verrecchia, F., et al. 2016, ApJL, 825, L4

[28] Ackermann, M., et al. 2016, ApJ, 823, L2

[29] Verrecchia, F., et al. 2017, ApJ, 847, L142 
[30] Verrecchia, F., et al. 2017, ApJ, 850, L142

\section{DISCUSSION}

WILLIAM N. BRANDT: Over the entire lifetime of AGILE, what is the most extreme variability you have observed from the Crab? What are the main physical implications of it?

CARLOTTA PITTORI: The brighest $\gamma$-ray flare from the Crab Nebula observed to date was the "super-flare" in mid-April 2011, reaching a record-breaking 1-day averaged flux of about a factor of 10 with respect to the steady state with risetime on a timescale below 1 day. These observations imply a very fast, localized and efficient acceleration mechanism of particles up to $10^{15} \mathrm{eV}$, challenging standard magnetohydro-dynamical (MHD) models, evidencing the possible role of plasma instabilities and impulsive particle acceleration in magnetic field reconnection.

FRANCO GIOVANNELLI's Comment: AGILE [NdR: observations leading to the] detection of a BH in a binary system with a Be star is very important, since such kind of systems are very rare in the Galaxy, as predicted by the "Scenario Machine" code of Vladimir Lipunov", and commented in our Workshops by Janusz Ziolkowski ${ }^{3}$.

DANIELE FARGION: May AGILE offer the sky map of Terrestrial Gamma-Ray Flashes (TGFs)? Indeed, as you said, thunderstorms may better amplify the trigger by upgoing tau-neutrino airshower, but their astrophysical map may (as I hope) be meaningfull (if astrophysical) or meaningless (if just geophysical).

CARLOTTA PITTORI: The AGILE-MCAL instrument, with its sub-millisecond trigger time scales, proved to be a very efficient instrument for TGF detection above $20 \mathrm{MeV}$, but it is has no imaging capability. However, as published by Martino Marisaldi in $2010^{4}$, the AGILE-GRID imaging data have been correlated with TGFs detected by the AGILE-MCAL providing the first precise direct localization of TGFs from space for a subset of events. All analyzed TGFs are compatible with a terrestrial production site closer to the satellite footprint. But I think that may be an idea to try to look at the AGILE-GRID TGF data by assuming a possible astrophysical origin of the trigger.

\footnotetext{
${ }^{2}$ V.M. Lipunov et al., Astronomy Reports, 2009, v. 53, no. 10, p. 915

${ }^{3}$ Ziolkowski, J., Proceedings of FRAPWS2014, NASA ADS Bibliografic Code: 2014frap.confE..43Z

${ }^{4}$ Marisaldi M. et al., 2010, Phys. Rev. Lett. 105, 128501
} 